\title{
Cortical Inputs and GABA Interneurons Imbalance Projection Neurons in the Striatum of Parkinsonian Rats
}

\author{
Nicolas Mallet, Bérangère Ballion, Catherine Le Moine, and François Gonon \\ Centre National de la Recherche Scientifique, Unité Mixte de Recherche 5541, Université Victor Segalen Bordeaux 2, 33076 Bordeaux, France
}

The striatum receives massive cortical excitatory inputs and is densely innervated by dopamine. Striatal projection neurons form either the direct or indirect pathways. Models of Parkinson's disease propose that dopaminergic degeneration imbalances both pathways, although direct electrophysiological evidence is lacking. Here, striatal neurons were identified by electrophysiological criteria and Neurobiotin labeling combined with either immunohistochemistry or in situ hybridization. Their spontaneous discharge activity and spike response to cortical stimulation were recorded in vivo in anesthetized rats rendered hemi-parkinsonian by 6-hydroxydopamine. We showed that striatonigral neurons (direct pathway) were inhibited whereas striatopallidal neurons (indirect pathway) were activated by dopaminergic lesion. We also identified, with antidromic stimulations, corticostriatal neurons that preferentially innervate striatonigral or striatopallidal neurons and showed that dopaminergic depletion selectively decreased the spontaneous activity of the former. Therefore, dopamine degeneration induces a cascade of imbalances that spread out of the basal ganglia and affect the whole basal ganglia-thalamo-cortical circuits.

Fast-spiking GABA interneurons provide potent feedforward inhibition of striatal projection neurons. We showed here that these interneurons narrowed the time window of the responses of projection neurons to cortical stimulation. In the dopamine-depleted striatum, because the intrinsic activity of these interneurons was not altered, their feedforward inhibition worsened the striatal imbalance. Indeed, the time window of the evoked responses was narrower for striatonigral neurons and wider for striatopallidal neurons. Therefore, after dopaminergic depletion, cortical inputs and GABA interneurons might imbalance striatal projection neurons and represent two novel nondopaminergic mechanisms that might secondarily contribute to the pathophysiology of Parkinson's disease.

Key words: basal ganglia; striatum; GABAergic; interneuron; dopamine; Parkinson's disease

\section{Introduction}

The degeneration of dopaminergic neurons is the primary cause of Parkinson's disease. According to pathophysiological models, imbalanced activities of projection neurons in the striatum are responsible for the hypokinesia associated with this disease and are caused by the lack of dopaminergic receptor stimulation (Albin et al., 1989; DeLong, 1990; Hikosaka et al., 2000; Bezard et al., 2001; Obeso et al., 2004). However, the possibility that nondopaminergic mechanisms might secondarily contribute to this striatal imbalance has not been considered in these models.

In the rat striatum, medium-sized spiny neurons (MSNs) represent $95 \%$ of the neurons. MSNs are GABAergic projection neurons and belong to two populations. Striatonigral neurons form the direct pathway to substantia nigra pars reticulata (SNR) and express substance $\mathrm{P}$ and dopaminergic $\mathrm{D}_{1}$ receptors. Striatopallidal neurons initiate the indirect pathway and express enkepha-

\footnotetext{
Received 0ct. 18, 2005; revised Jan. 31, 2006; accepted Feb. 24, 2006.

This work was supported by the Centre National de la Recherche Scientifique, the Université Victor Segalen Bordeaux 2, the Parkinson Disease Foundation, and the Michael J. Fox Foundation for Parkinson's Research. We thank Drs. Thomas Boraud, Patricio O'Donnell, and Kuei Tseng for thoughtful discussion and critical reading of this manuscript.

Correspondence should be addressed to François Gonon, Centre National de la Recherche Scientifique, Unité Mixte de Recherche 5541, Boîte postale 28, Université Victor Segalen Bordeaux 2, 33076 Bordeaux, France. E-mail: francois.gonon@umr5541.u-bordeaux2.fr.

DOI:10.1523/JNEUROSCI.4439-05.2006

Copyright $\odot 2006$ Society for Neuroscience $\quad 0270-6474 / 06 / 263875-10 \$ 15.00 / 0$
}

lin (ENK) and $\mathrm{D}_{2}$ receptors (Le Moine and Bloch, 1995; Gerfen and Wilson, 1996). Models mentioned above propose that dopaminergic depletion disinhibits striatopallidal neurons and removes the facilitatory influence of $\mathrm{D}_{1}$ stimulation on striatonigral neurons. However, this striatal imbalance is only documented by anatomo-functional evidence (Gerfen, 2000; Obeso et al., 2000). We developed in vivo electrophysiological recordings of identified neurons in the striatum of rats rendered hemi-parkinsonian by unilateral 6-hydroxydopamine (6-OHDA) injection to directly show that dopaminergic depletion imbalances MSN activities.

In urethane-anesthetized rats, MSNs synchronously oscillate between a hyperpolarized, quiescent state and a depolarized state, which enables firing to occur. The depolarized state is caused by a maintained barrage of synaptic excitation originating from the cortex, although the membrane potential achieved during that state is also determined by intrinsic voltage-dependent potassium currents (Wilson and Kawaguchi, 1996). Striatonigral MSNs are preferentially innervated by cortical neurons that project into telencephalon (IT-type), including both striatum, whereas striatopallidal MSNs receive greater input from ipsilateral cortical neurons that send their main axons into pyramidal tract (PT-type) (Lei et al., 2004). We used antidromic stimulations of both striatum to identify IT-type and PT-type neurons and we tested whether the imbalance between striatonigral and 
striatopallidal neurons caused by dopaminergic depletion is associated with imbalanced activities of IT-type and PT-type neurons.

Although striatal GABAergic interneurons only represent $1-2 \%$ of the neuronal population (Kawaguchi et al., 1995), in vitro electrophysiological studies showed that they represent the main source of GABA inhibition of MSNs (Plenz and Kitai, 1998; Koos and Tepper, 1999; Koos et al., 2004). Of these interneurons, the most numerous, especially in the lateral striatum, are positive for parvalbumin and are called fast-spiking interneurons (FSIs) because of their brief spike duration (Kita et al., 1990; Kawaguchi, 1993; Kawaguchi et al., 1995). Striatonigral and striatopallidal MSNs equally receive from FSIs a potent feedforward inhibition that filters striatal integration of cortical inputs (Mallet et al., 2005). In vitro, FSIs are depolarized by dopamine (DA) via dopaminergic receptors of the $\mathrm{D}_{1}$ type (Bracci et al., 2002; Centonze et al., 2003). Moreover, dopaminergic depletion reduces the immediate-early gene response of parvalbumin-positive neurons to cortical stimulation (Trevitt et al., 2005). Therefore, dopaminergic depletion may release MSNs from normal feedforward inhibition. We have tested whether striatal FSIs contribute to the striatal imbalance in hemi-parkinsonian rats.

\section{Materials and Methods}

6-Hydroxydopamine injections in the medial forebrain bundle. Experiments were performed in vivo in accordance with French (87-848, Ministère de l'Agriculture et de la Forêt) and European Economic Community (86-6091 EEC) guidelines for the care of laboratory animals and were approved by the Ethical Committee of Centre National de la Recherche Scientifique, Région Aquitaine. Adult Sprague Dawley rats $(240-280 \mathrm{~g})$ were anesthetized with ketamine hydrochloride $(75 \mathrm{mg} / \mathrm{kg}$, i.p.) and xylazine hydrochloride $(10 \mathrm{mg} / \mathrm{kg}$, i.p.). Rats were fixed on a stereotaxic frame and received desipramine hydrochloride $(25 \mathrm{mg} / \mathrm{kg}$, s.c.). The localization of the medial forebrain bundle was checked with a carbon fiber electrode $(1.6 \mathrm{~mm}$ lateral to medial line and 3.8 caudal to bregma) coupled with voltammetry to detect 3,4-dihydroxyphenylacetic acid (DOPAC) synthesized by dopaminergic axons (Svenningsson et al., 1999). 6-OHDA (3 $\mu \mathrm{g} / \mu \mathrm{l})$ was dissolved in saline solution containing ascorbic acid ( $1 \mathrm{~mm}$ ) and injected with air pressure using a glass pipette (Svenningsson et al., 1999). Four injections ( $0.5 \mu \mathrm{l} / 1 \mathrm{~min}$ each) separated by $250 \mu \mathrm{m}$ vertical intervals were performed above and below the point at which the DOPAC level was maximum. Forty-seven rats were injected with the toxin, and 37 rats were injected with vehicle.

Electrophysiological recordings. Three to 4 weeks after cerebral injections, rats were anesthetized with urethane, and single striatal neurons were extracellularly recorded from the rostrolateral striatum and from the orofacial motor cortex, as described previously (Mallet et al., 2005). Briefly, glass pipettes (13-24 M $\Omega$ ) simultaneously recorded spike discharges and local field potentials. Antidromic stimulation of SNR was used to identify striatonigral neurons (Mallet et al., 2005). Concentric bipolar electrodes were implanted in the ipsilateral and contralateral striatum as described previously (Mallet et al., 2005) to identify corticostriatal neurons on the basis of their antidromic responses. Antidromic responses fulfilled the following criteria: (1) constant latency of spike response, (2) all-or-none property of the spike response when the strength of the stimulation was adjusted just above, or just below, threshold, and (3) collision of the antidromic spikes with orthodromic spikes. Because most striatal neurons are silent, the orthodromic spike required for the collision test was evoked by cortical stimulation, and the SNR stimulation was triggered by the spike evoked by cortical stimulation with a delay of 30 or $3 \mathrm{~ms}$, alternately. Juxtacellular injection of Neurobiotin was used to label striatal neurons (Mallet et al., 2005).

Histological methods. After electrophysiological recordings, rats were perfused through the heart with $30 \mathrm{ml}$ of saline $(9 \% \mathrm{NaCl})$ and then with $200 \mathrm{ml}$ of $4 \%$ paraformaldehyde (PFA). After perfusion, brains were dissected and kept overnight in 4\% PFA. Vibratome sections $(60 \mu \mathrm{m}$ thick) were collected in PBS. Double immunohistochemistry was used to identify FSIs by the codetection of Neurobiotin and parvalbumin (Mallet et al., 2005). Double labeling for Neurobiotin and ENK mRNA was used to identify striatopallidal neurons using a procedure adapted from Le Moine and Bloch (1995) and Svenningsson et al. (1997). Briefly, freefloating sections were hybridized with a digoxigenin-labeled ENK cRNA probe. After posthybridization rinsing, sections were then transferred in PBS for the processing of Neurobiotin and digoxigenin detections and rinsed 10 min twice in PBS/0.3\% Triton X-100. Sections were first incubated with FITC-conjugated streptavidin (SA) diluted 1:500 in PBS/0.3\% Triton X-100 for Neurobiotin visualization. After $4 \mathrm{~h}$ at room temperature, FITC-SA was removed and replaced by a peroxidase-conjugated anti-digoxigenin antibody (Roche Products, Welwyn Garden City, UK). The antibody was incubated $2 \mathrm{~h}$ at room temperature and then overnight at $4^{\circ} \mathrm{C}$. After rinsing, the sections were incubated with biotinyl tyramide (1:50; PerkinElmer, Wellesley, MA) for $10 \mathrm{~min}$ and then rinsed in PBS (10 min for three times). The signal was then visualized after $30 \mathrm{~min}$ incubation with SA conjugated with Alexa Fluor 568 (Invitrogen, Carlsbad, CA), rinsing again in PBS three times for $10 \mathrm{~min}$, and mounting in Vectashield (Vector Laboratories, Burlingame, CA).

Data analysis. Field potentials recorded in the cortex and striatum during periods of continuous slow waves (SWs) lasting for at least 2 min were analyzed with fast Fourier transform: the power spectrum was built from each recording by the Spike2 program (Cambridge Electronic Design, Cambridge, UK) (Mallet et al., 2005), as illustrated in Figure 3G. This analysis was used to assess the quality of the SWs. Indeed, when a period of SWs was significantly contaminated with brief episodes of desynchronization, the maximum of the power spectrum was found below $0.01 \mathrm{mV}^{2}$, and the corresponding recording was not considered further. SWs simultaneously recorded in the cortex and striatum were compared with cross-correlation displayed by Spike2, with the cortical wave as reference wave, from recording periods of $30 \mathrm{~s}$. From these crosscorrelograms, two parameters were measured: the maximum of the correlation coefficient and the time interval between that maximum and time 0 . The discharge activity of single neurons was analyzed with interspike interval (ISI) histograms built by Spike2 with a bin width of $2 \mathrm{~ms}$. The temporal relationship between slow waves and spike discharges was shown for every individual neuron by averaging, using Spike2, the field potentials recorded from -1 to +1 s around every spike as illustrated in Figure $3 D$. Continuous periods of slow waves, during which at least 10 spikes were recorded, were used to build these averaged field potentials. From these averaged recordings, the time interval between their maximum and time 0 (corresponding to every spike) was measured for every single neuron. Paired and unpaired $t$ tests were used for statistical comparisons.

\section{Results}

\section{Identification of striatal neurons after dopaminergic lesion}

In all 47 rats injected with 6-OHDA, the number of cells immunoreactive for tyrosine hydroxylase $(\mathrm{TH})$ in the substantia nigra pars compacta was decreased by at least $95 \%$ compared with the side contralateral to 6-OHDA injection (data not shown). This resulted in a complete loss of $\mathrm{TH}$-positive innervation in the lateral striatum (supplemental Fig. 1, available at www.jneurosci. org as supplemental material). However, in most cases, this innervation was still partly preserved in the nucleus accumbens. In four rats, immunohistology for $\mathrm{TH}$ and for parvalbumin were combined (supplemental Fig. 1, available at www.jneurosci.org as supplemental material). As reported previously (Trevitt et al., 2005), dopaminergic depletion did not affect the number of parvalbumin-positive cells in the striatum. The present study was performed, as described previously (Mallet et al., 2005), in the rostrolateral striatum because the density of parvalbuminpositive cells is higher in this region than in the medial striatum (supplemental Fig. 1, available at www.jneurosci.org as supplemental material).

Striatonigral neurons were identified by their antidromic responses to SNR stimulation (Fig. 1A) and were named $\mathrm{SNR}^{+}(31$ 

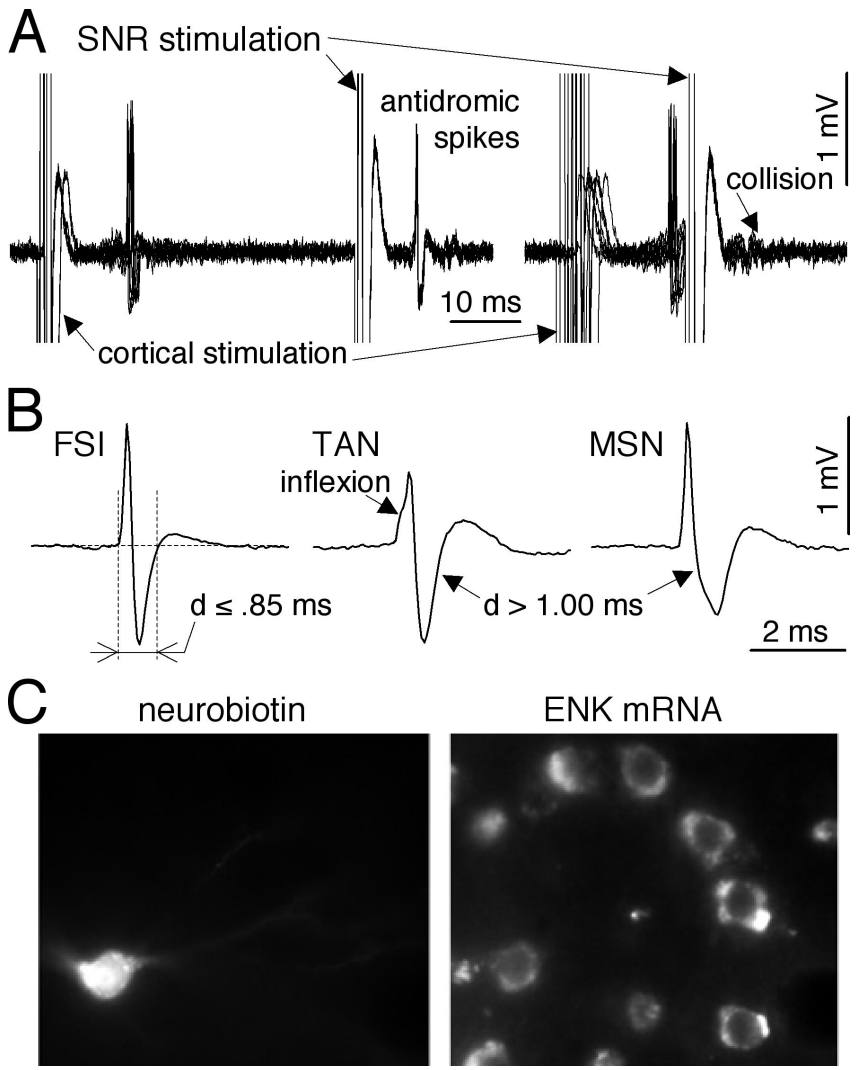

Figure 1. Neuronal identification in the DA-denervated striatum. $\boldsymbol{A}$, Stimulation of the SNR was triggered by an orthodromic spike evoked by cortical stimulation with a time interval of 30 $\mathrm{ms}$ (left) or $3 \mathrm{~ms}$ (right). Recordings show the superimposition of 10 successive responses to SNR stimulation in both conditions. Notice the fixed latency of the antidromic spikes (left) and the collision of the orthodromic spike with the antidromic one at short intervals (right). Only neurons that fulfilled the three criteria of an antidromic response (see Materials and Methods) were considered to be striatonigral and called SNR ${ }^{+}$. $\boldsymbol{B}$, Spike waveforms of three types of striatal neurons recorded in the same experiment. Traces show the averaging of 20 successive spikes. FSIs were distinguished from all other striatal neurons by their briefer spike duration. $\boldsymbol{C}$, Identification of striatopallidal neurons. This typical neuron was presumed to be striatopallidal because it did not exhibit any antidromic response to SNR stimulation and did not exhibit the electrophysiological characteristics of FSIs or cholinergic interneurons (see Results). The neuron was injected with Neurobiotin, which was revealed by immunohistochemistry (left). In situ hybridization shows that this neuron was positive for ENK mRNA, a recognized feature of striatopallidal neurons (right).

neurons in sham-injected rats and 32 in 6-OHDA-lesioned rats). In intact rats (Mallet et al., 2005) and in sham-injected rats, $\mathrm{SNR}^{+}$neurons were detected by their response to cortical stimulation and then identified by antidromic stimulation. In contrast, in DA-denervated striatum, we often observed striatal neurons, which were completely silent and did not respond to cortical stimulation, although they did respond to SNR stimulation in an antidromic way (constant latency of spike response and all-or-none property of the spike response). However, because it was not possible to perform the collision test, these neurons were not studied further. Therefore, it is likely that the $32 \mathrm{SNR}^{+}$studied in 6-OHDA-lesioned rats represented a subpopulation of striatonigral MSNs that were more responsive to cortical stimulation than the whole population.

FSIs were identified by their spike duration, which was briefer than that of all other striatal neurons in DA-denervated striatum (13 FSIs) (Fig. 1B), in sham-injected rats (15 FSIs; data not shown), and in intact rats (Mallet et al., 2005). Among the 13 FSIs recorded in DA-denervated striatum, four were injected with
Neurobiotin. As in intact rats (Mallet et al., 2005), all four injected FSIs were also positive for parvalbumin (data not shown). Tonically active neurons (TANs) were identified by their unique, regularly spaced, spontaneous activity between 2 and $6 \mathrm{~Hz}$. Moreover, their spike exhibited an inflection point in the first phase (Fig. $1 B$ ). TANs were not further studied here. Finally, neurons that did not belong to the three groups described above and that did not exhibit antidromic responses to SNR stimulations were named SNR ${ }^{-}$(66 in 6-OHDA-lesioned striatum and 42 in shaminjected rats). It is likely that the vast majority of SNR neurons were striatopallidal, as discussed previously (Mallet et al., 2005). To further support this view, representative SNR ${ }^{-}$neurons were injected with Neurobiotin. All four neurons injected in shamoperated rats and all but one of the seven neurons injected in DA-denervated striatum were also positive for ENK mRNA (Fig. 1C). Control experiments showed that all five $\mathrm{SNR}^{+}$neurons injected with Neurobiotin in intact rats were negative for ENK mRNA (data not shown).

\section{Spontaneous activity of identified striatal neurons}

Cortical and striatal field potentials exhibited two spontaneous states in rats anesthetized with urethane: a dominant state, characterized by synchronous SWs of large amplitudes at $\sim 1 \mathrm{~Hz}$ $\left(\mathrm{SW}^{+}\right.$state) and periods of absence of slow waves ( $\mathrm{SW}^{-}$state) (Mallet et al., 2005). Both patterns were also observed in the cortex and striatum ipsilateral to 6-OHDA injections (see examples of SWs in Fig. 2A). We showed in intact rats that the striatal SW closely follows the cortical SW with a delay of $11 \pm 8 \mathrm{~ms}$ (mean \pm SD) (Mallet et al., 2005). This relationship was not affected by dopaminergic depletion. Indeed, cross-correlograms between striatal and cortical SWs were studied in $10 \mathrm{DA}$-depleted rats and exhibited a maximal coefficient close to $1(0.88 \pm 0.04$, mean $\pm \mathrm{SD} ; 20$ determinations in 20 distinct striatal sites). The striatal SW appeared with a delay of $14.5 \pm 7$ ms (mean \pm SD). For each period of simultaneous recording of spontaneous discharge activity and of field potentials, the striatal SWs were analyzed with fast Fourier transform, and the resulting power spectrum always exhibited a single prominent peak at $\sim 0.85 \mathrm{~Hz}$ whose amplitude and frequency were not significantly affected by dopaminergic depletion (data not shown).

During $\mathrm{SW}^{+}$state, MSNs and FSIs spontaneously discharge in synchrony with SWs closely to the maximum of the field potential (Mallet et al., 2005), which corresponds to the depolarized state of MSNs (Tseng et al., 2001). This discharge pattern was also observed in the DA-denervated striatum as exemplified in Figure $2, A$ and $B$. More precisely, in sham-operated rats, $\mathrm{SNR}^{-}$neurons discharged before the maximum of the striatal SW (Fig. 2C). The same pattern was observed in intact rats and was thought to be attributable to the inhibition of MSNs by FSIs: MSNs might become silent during the depolarized state when FSIs reach their maximal activity (Mallet et al., 2005). In 6-OHDA-lesioned rats, $\mathrm{SNR}^{-}$neurons tended to discharge more closely to the maximum of the SW and with a wider distribution, although the difference did not reach statistical significance. This is consistent with the view that, in 6-OHDA-lesioned rats, striatopallidal neurons tend to escape from feedforward inhibition by FSIs. Dopaminergic depletion strongly depressed the mean discharge rate of $\mathrm{SNR}^{+}$neurons and enhanced that of $\mathrm{SNR}^{-}$neurons but did not affect that of FSIs (Fig. 2D). When SWs were disrupted by a tail pinch, all 13 FSIs recorded in 6-OHDA-lesioned rats responded by a burst of action potentials, and all spontaneously active MSNs (29 $\mathrm{SNR}^{-}$and one $\mathrm{SNR}^{+}$) were silenced (data not shown), as shown previously in intact rats (Mallet et al., 2005). 

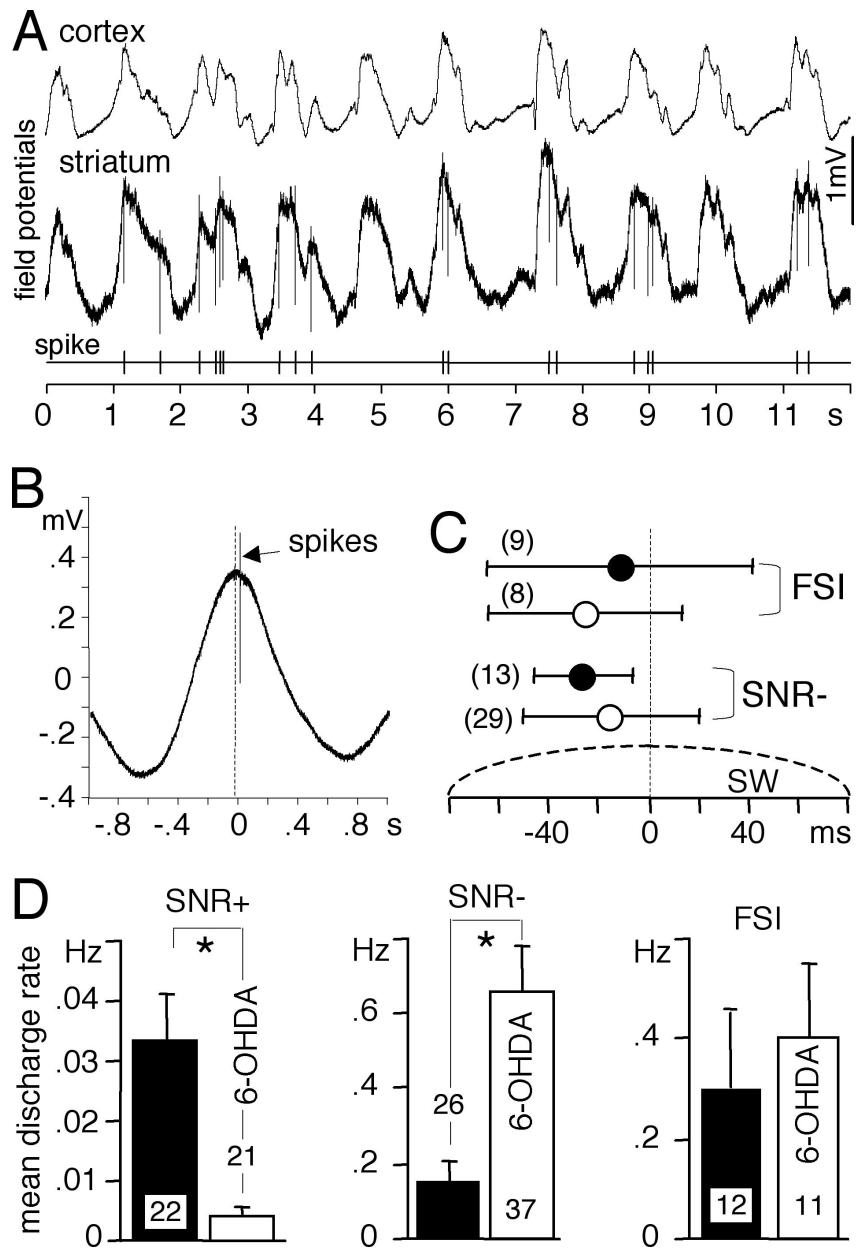

Figure 2. Spontaneous discharge activity of striatal neurons during slow waves. $\boldsymbol{A}$, Cortical and striatal field potentials were recorded simultaneously with the discharge activity of one $\mathrm{SNR}^{-}$neuron in a 6-OHDA-lesioned rat. Notice that the striatal field potential closely followed the cortical one and that action potentials only occurred during the maximum of the field potentials. $\boldsymbol{B}$, Temporal relationship between discharge activity and striatal slow waves. The discharge activity (174 spikes) was recorded simultaneously with the field potential during a continuous period of SW ${ }^{+}$state $(267 \mathrm{~s}$ ). Sections of striatal field potential recordings centered on every spike were averaged together. On average, this SNR ${ }^{-}$neuron recorded in a 6-OHDAlesioned rat discharged after the maximum of the slow waves. $C$, Temporal relationship between the striatal SW and the discharge activity of FSI and presumed striatopallidal neurons $\left(\mathrm{SNR}^{-}\right)$recorded in sham-operated rats (filled circles) and in 6-OHDA lesioned rats (open cir(les). The number of neurons studied is indicated in parentheses. Error bars indicate mean \pm SD. All neurons discharged closely to the maximum of the striatal slow wave. $\boldsymbol{D}$, Mean discharge activities of striatonigral neurons ( $\mathrm{SNR}^{+}$), presumed striatopallidal neurons (SNR ${ }^{-}$), and FSIs compared in sham-operated rats (filled bars) and in 6-OHDA-lesioned rats (open bars). Bar histograms indicate mean \pm SEM (unpaired $t$ test, ${ }^{*} p \leq 0.0019$ ). The number of neurons studied is indicated inside or above bars. The dopaminergic depletion strongly decreased the discharge activity of striatonigral neurons and enhanced that of striatopallidal neurons.

\section{Spontaneous activity of identified corticostriatal neurons}

The rostrolateral striatum is innervated by the orofacial motor cortex (Deniau et al., 1996). Because IT-type neurons project into both striatum, whereas PT-type neurons only innervate the ipsilateral striatum (Lei et al., 2004), antidromic stimulations of the ipsilateral and contralateral striatum were used to discriminate between IT-type and PT-type corticostriatal neurons of the orofacial cortex (Fig. 3A). Cortical neurons exhibiting antidromic responses from both striatum were presumed to be of the ITtype, whereas those responding only to ipsilateral stimulation were likely to be of the PT-type (Fig. $3 B$ ). The latency of the antidromic responses to contralateral stimulations ranged from 6.8 to $20.1 \mathrm{~ms}(11.0 \pm 3.2 \mathrm{~ms}$, mean $\pm \mathrm{SD} ; n=41)$. This latency distribution is in agreement with a previous study (Cowan and Wilson, 1994). In 2 of 17 experiments, shorter latencies (range of $2.8-5.2 \mathrm{~ms}$ ) were observed for five neurons. In both experiments, the stimulating electrode was located in the contralateral striatum but too close to the white matter. Therefore, these neurons were not further considered because it is likely that they corresponded to corticocortical neurons and not to corticostriatal neurons of the IT-type, as suggested by Cowan and Wilson (1994).

As observed previously (Mallet et al., 2005), during SW ${ }^{+}$ state, both types synchronously discharged during the maximum of the SWs in both sham-operated rats and DA-denervated rats (Fig. 3C,D). Dopaminergic depletion did not alter this temporal relationship (Table 1). Dopaminergic depletion strongly depressed the mean discharge rate of presumed IT-type cortical neurons and only slightly decreased that of presumed PT-type neurons, although this latter effect did not reach statistical significance $(p=0.31)$ (Fig. $3 E)$. Overall, in sham-operated rats, both neuronal types exhibited similar activities, and dopaminergic depletion severally upset this equilibrium. Most IT-type and PTtype neurons often discharged with double spikes as illustrated in Figure $3 C$. Triple spikes were more rarely observed. This bursting activity was analyzed with interspike interval histograms (Fig. $3 F)$. Regarding IT-type neurons, the dopaminergic depletion strongly decreased the occurrence of longer ISIs, whereas that of double spikes was less affected (Fig. 3F, Table 1). The reverse effects were observed regarding PT-type neurons (Table 1).

Because dopaminergic depletion strongly decreased the discharge activity of IT-type neurons and because SWs of cortical field potential are generated by the synchronous discharge activity of cortical neurons, this depletion might quantitatively affect SWs. Fast Fourier analysis of cortical field potentials recorded during $\mathrm{SW}^{+}$state showed a single prominent peak at $\sim 0.85 \mathrm{~Hz}$ (Fig. 3G). This dominant frequency was not affected by dopaminergic depletion, whereas the maximal amplitude of the peak was significantly decreased (Fig. 3G, Table 2). However, taking into account that the power spectrum is calculated from the square of the SWs, this decrease corresponds to a minor alteration in the amplitude of the SWs. This alteration was not caused by differences in the depth of anesthesia because sham-operated and 6 -OHDA-lesioned rats received the same amount of urethane (Table 2) and remained most of the time in the dominant $\mathrm{SW}^{+}$ state, except when it was disrupted by tail pinch.

During $\mathrm{SW}^{-}$state, either induced by tail pinch or spontaneously occurring, cortical neurons exhibited, on average, similar discharge rates compared with $\mathrm{SW}^{+}$state in 6-OHDA-lesioned rats, in sham-operated rats (data not shown), and in intact rats (Mallet et al., 2005). Therefore, the significant decrease induced by dopaminergic depletion in the spontaneous activity of presumed IT-type neurons recorded during $\mathrm{SW}^{+}$state (Fig. 3E) was also observed during $\mathrm{SW}^{-}$state (data not shown; 13 neurons in sham-operated rats and 12 neurons in 6-OHDA-lesioned rats; unpaired $t$ test, $p=0.022$ ).

\section{Spike responses of striatal neurons to paired cortical stimulation}

We used a protocol of paired cortical stimulation at $100 \mathrm{~ms}$ time interval, which reveals feedforward inhibition of MSNs by FSIs (Mallet et al., 2005). The response of striatal neurons to cortical stimulation was investigated here only during $\mathrm{SW}^{-}$state, whether spontaneously occurring or induced by tail pinch. In fact, the response probability strongly depends on the occurrence 

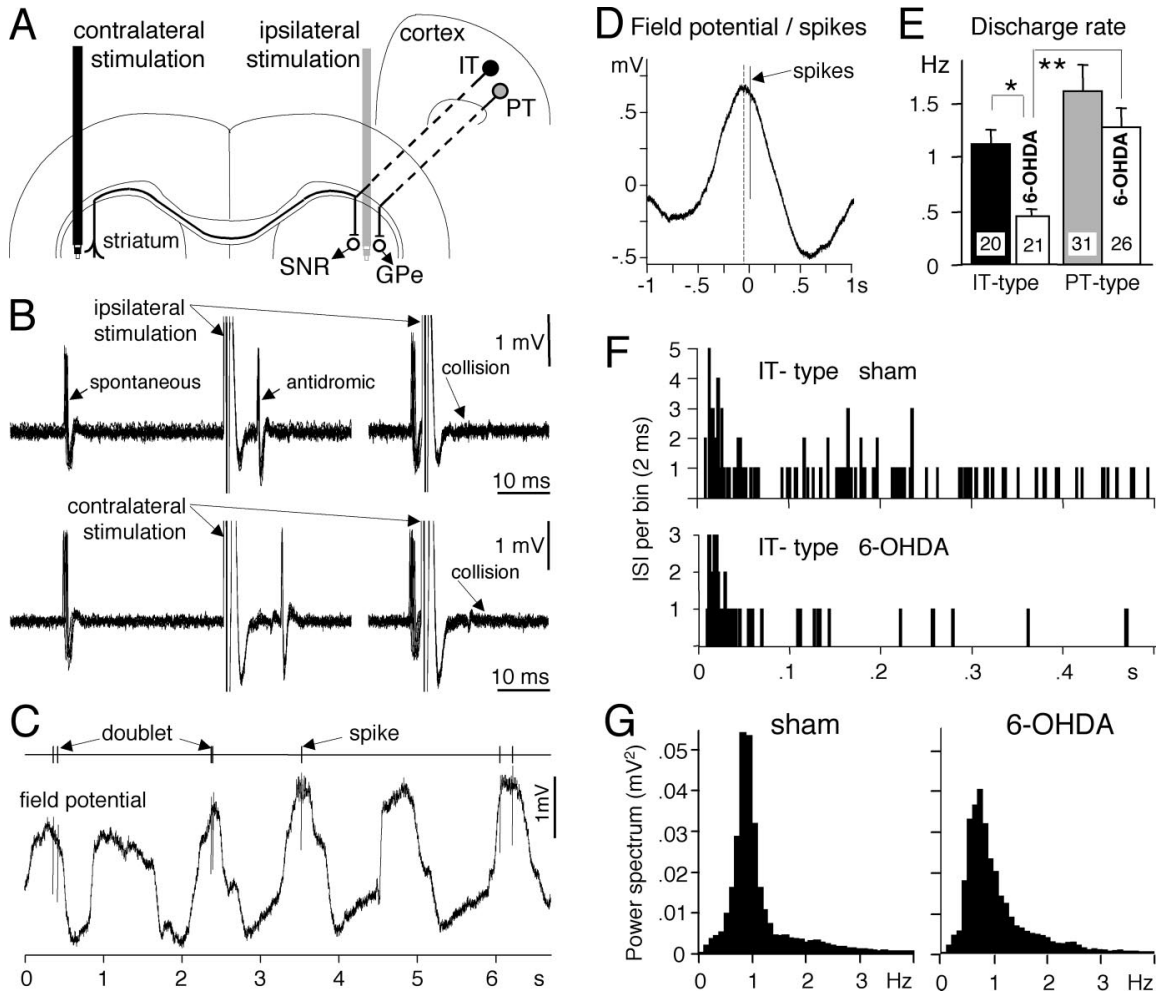

Figure 3. Identification and spontaneous discharge activity of corticostriatal neurons during slow waves. $\boldsymbol{A}$, Striatonigral MSNs are preferentially innervated by cortical neurons that project into telencephalon (IT-type), whereas striatopallidal MSNs receive greater input from those that send their main axon into pyramidal tract (PT-type) (Lei et al., 2004). Because IT-type neurons project into both striatum whereas PT-type neurons only innervate the ipsilateral striatum, the antidromic stimulation of both striatum can be used to discriminate between IT-type and PT-type neurons. $\boldsymbol{B}$, Example of an IT-type neuron exhibiting antidromic responses to stimulation of both striatum. Striatal stimulation was triggered by spontaneous spikes with a time interval of $30 \mathrm{~ms}$ (left) or $3 \mathrm{~ms}$ (right). Recordings show the superimposition of 10 successive responses to striatal stimulation in both conditions. Notice the fixed latency of the antidromic spikes (left) and the collision of the spontaneous spike with the antidromic one at short intervals (right). Only neurons that fulfilled the three criteria (see Materials and Methods) of a double antidromic response from both striatum were considered to be of the IT-type. Neurons that only exhibited an antidromic response to ipsilateral stimulation were presumed to be of the PT-type. Cortical neurons that did not show any antidromic response were discarded. $C$, Simultaneous recording of the cortical field potential and of the discharge activity of the IT-type neuron identified in $B$, recorded in a 6-0HDAlesioned rat. During $\mathrm{SW}^{+}$state, all corticostriatal neurons discharged during the maximum of the SWs. $\boldsymbol{D}$, Temporal relationship between discharge activity and slow waves. The discharge activity (75 spikes) was recorded simultaneously with the field potential during a continuous period of SW ${ }^{+}$state $(388 \mathrm{~s}$ ). Sections of field potential recordings centered on every spike were averaged together. On average, this IT-type neuron recorded in a 6-0HDA-lesioned rat discharged after the maximum of the slow waves. $\boldsymbol{E}_{\text {, }}$ Spontaneous discharge rate of corticostriatal neurons. Bar histograms indicate mean \pm SEM (unpaired $t$ test, ${ }^{*} p=0.0001,{ }^{* *} p=$ 0.0002 ; other differences $p \geq 0.14$ ). The number of neurons studied is indicated inside bars. These neurons were recorded from nine sham-lesioned rats (filled bars) and from eight 6-0HDA-lesioned rats (open bars). $\boldsymbol{F}$, The discharge activity of two IT-type neurons was analyzed with ISI histograms (2 ms bin). G, Power spectrum built from field potentials recorded in the cortex of one sham-lesioned rat and of one 6-OHDA-lesioned rat. Field potential was recorded during periods of continuous SW ${ }^{+}$state lasting for 2-10 min. Dopaminergic depletion strongly depressed the mean discharge rate of IT-type neurons.

Table 1. Spontaneous activity of corticostriatal neurons during slow waves

\begin{tabular}{|c|c|c|c|c|}
\hline & \multicolumn{2}{|l|}{ Sham (9 rats) } & \multicolumn{2}{|l|}{ 6-OHDA (8 rats) } \\
\hline & IT-type $(n=20)$ & PT- type $(n=31)$ & IT-type $(n=21)$ & PT-type $(n=26)$ \\
\hline Spikes in relation to SW (ms) & $-4.4 \pm 22.8$ & $-3.1 \pm 27.2$ & $-14.5 \pm 37.3$ & $6.2 \pm 35.7$ \\
\hline Bursting (\%) & $49.7 \pm 18.9$ & $52.1 \pm 21.2$ & $63.0 \pm 13.3$ & $40.9 \pm 18.9$ \\
\hline$($ ISI $<100 \mathrm{~ms}) /(I S I<500 \mathrm{~ms})$ & & & $p=0.016$ & $p=0.043$ \\
\hline$(I S I<100$ ms)/(all ISI) (\%) & $22.1 \pm 10.1$ & $25.2 \pm 13.6$ & $22.5 \pm 12.1$ & $19.9 \pm 13.7$ \\
\hline
\end{tabular}

Data were obtained from the spike analysis described in Figure 3. Corticostriatal neurons always discharged in close relationship with the maximum of the slow wave, as illustrated in Figure 3D. Negative values mean that spikes occurred before this maximum. Values are expressed as mean \pm SD. Data from shamoperated and 6-OHDA-treated rats were compared with unpaired $t$ test.

of the stimulation during the various phases of SWs, and this variability further complicated analysis and interpretation of the data (Mallet et al., 2005). In intact rats, FSIs respond to paired cortical stimulations with unique characteristics: higher proba-

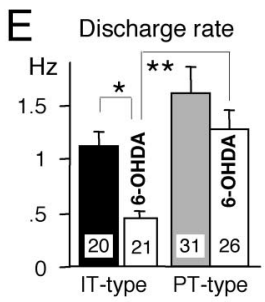

bility of the response to the first pulse in a pair and burst responses to suprathreshold stimulations (Mallet et al., 2005). Both characteristics were observed in shamoperated rats and remained unaffected by dopaminergic depletion (Fig. 4A-C). Moreover, the stimulating current required to evoke a $50 \%$ probability of the response to the first pulse was only slightly enhanced in 6-OHDA-lesioned rats (Fig. $4 A$ ), but this increase did not reach statistical significance. As reported in intact rats (Mallet et al., 2005), the latency of the spike responses to the first pulse decreased with the increase of spike probability (Fig. $4 B$ ). This latency was not significantly affected by dopaminergic depletion either when the stimulation current was adjusted to evoke spike responses with a $50 \%$ probability (Fig. $4 \mathrm{~A}$ ) or when this current was increased to obtain a $90 \%$ spike probability $(8.9 \pm 0.4 \mathrm{~ms}$ in 13 sham-lesioned rats and $7.8 \pm 0.5 \mathrm{~ms}$ in 126 -OHDA-lesioned rats, mean \pm SEM).

In intact rats (Mallet et al., 2005) and in sham-operated rats, a few SNR ${ }^{+}$( 2 of 27 ) and $\mathrm{SNR}^{-}$( 6 of 26) neurons responded to paired cortical stimulation with a higher probability to the first than to the second pulse (Fig. 5B). Dopaminergic depletion did not alter this response pattern regarding $\mathrm{SNR}^{+}$neurons ( 2 of 25 neurons were more responsive to the first pulse) but reversed it regarding $\mathrm{SNR}^{-}$neurons (21 of 38 neurons were more responsive to the first pulse) (Fig. 5A,B). Moreover, the stimulating current required to reach a $50 \%$ probability of spike responses, evoked by either the first or second pulse, was similar for $\mathrm{SNR}^{+}$and $\mathrm{SNR}^{-}$neurons in intact rats (Mallet et al., 2005) and in sham-operated rats (Fig. 5C). This current was much higher than that needed for FSIs (compare Figs. 4A, 5C) $(i=312 \pm 31 \mu \mathrm{A}$ for the $27 \mathrm{SNR}^{+} ; i=342 \pm 26 \mu \mathrm{A}$ for the $26 \mathrm{SNR}^{-} ; i=118 \pm 19 \mu \mathrm{A}$ for the $13 \mathrm{FSIs}$; mean \pm SEM). Dopaminergic depletion resulted in an increase of this current regarding $\mathrm{SNR}^{+}$neurons and a decrease regarding $\mathrm{SNR}^{-}$neurons (Fig. $5 C$ ). In each neuronal group, this $50 \%$ probability current tended to be lower regarding neurons that were more responsive to the first pulse. Thus, in 6-OHDA-lesioned rats, the $21 \mathrm{SNR}^{-}$neurons, which were more responsive to the first pulse, needed a current $(178 \pm 22 \mu \mathrm{A}$, mean \pm SEM $)$ that was slightly, but not significantly, higher than that needed for FSIs $(137 \pm 19 \mu \mathrm{A}$, mean \pm SEM; $n=12$ ). Therefore, whereas FSIs were much more responsive to cortical stimulation than MSNs in control conditions, this was no longer the case regarding a majority of striatopallidal neurons in 6-OHDA-lesioned rats. 
Table 2. Cortical field potential during slow waves

\begin{tabular}{lll}
\hline Peak of the power spectrum & Sham (9 rats) & 6 -OHDA (8 rats) \\
\hline Number of recording sites & 51 & 47 \\
Maximal amplitude $\left(\mathrm{mV}^{2}\right)$ & $0.0501 \pm 0.0243$ & $0.0356 \pm 0.0226^{*}$ \\
Frequency $(\mathrm{Hz})$ & $0.82 \pm 0.14$ & $0.88 \pm 0.31$ \\
Urethane $(\mathrm{g} / \mathrm{kg})$ & $1.57 \pm 0.13$ & $1.63 \pm 0.14$ \\
\hline
\end{tabular}

Data were obtained from power spectra, as illustrated in Figure $3 \mathrm{G}$. In each experiment, the cortical field potential was recorded from 4-12 distinct cortical sites for time periods lasting for 2-10 min of continuous SW. The powe spectrum was constructed from each recording and always exhibited only one prominent peak at $\sim 0.85 \mathrm{~Hz}$. The maximal amplitude and frequency of each peak were measured. Values are expressed as mean $\pm S D$. Data from sham-operated and 6-OHDA-treated rats were compared with unpaired $t$ test. ${ }^{*} p=0.003$; other differences $p \geq$ 0.22

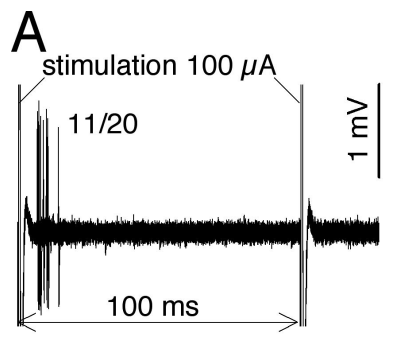

current $(\mu \mathrm{A}) \quad$ latency $(\mathrm{ms})$

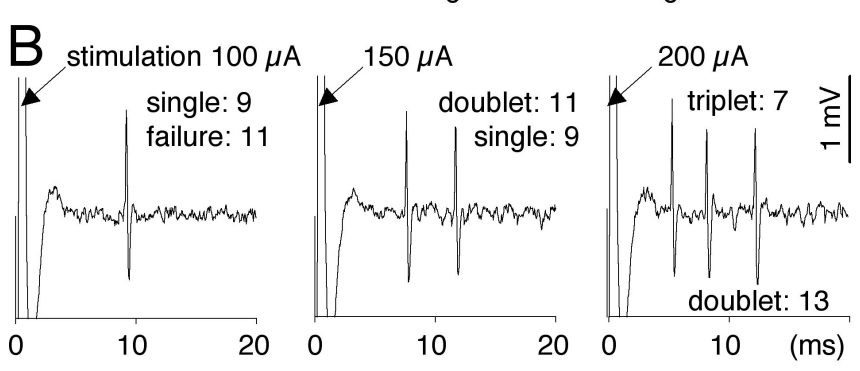

C

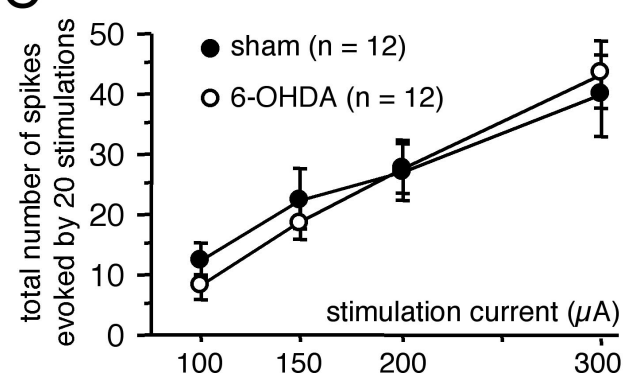

Figure 4. Effect of dopaminergic depletion on the responses of FSIs to cortical stimulations. $A$, Pairs of cortical stimulation (100 ms interval) were applied 20 times every $3 \mathrm{~s}$. For each individual FSI, the stimulating current was adjusted by preliminary tests so that the spike response to the first pulse reached a probability close to $50 \%$. The overlay of 20 consecutive traces, which were recorded from one FSI in a 6-OHDA-lesioned rat, is shown (left). Bar histograms (right) indicate that the stimulating current required for a response probability of $50 \%$ and the latency of the spike response to the first pulse were not significantly affected by dopaminergic depletion (open bars) compared with control (filled bars). Bar histograms indicate mean \pm SEM, and the number of neurons studied is indicated inside bars. $\boldsymbol{B}$, Examples of burst responses to series of 20 consecutive stimulations applied every $3 \mathrm{~s}$ at threshold and suprathreshold currents, which were evoked in one FSI recorded in a 6-OHDA-lesioned rat. C, Cumulative spike responses (mean \pm SEM) of FSIs to four series of 20 consecutive stimulations at increasing currents. Dopaminergic depletion did not significantly affect FSI responses.

\section{Latency of spike responses to cortical stimulation}

Local picrotoxin injection was used to reveal the potent feedforward inhibition of MSNs by FSIs (Mallet et al., 2005). As shown previously in the hippocampus (Pouille and Scanziani, 2001) and in the cerebellum (Mittmann et al., 2005), we observed that feedforward inhibition by GABA interneurons narrowed the time window of the response of MSNs to cortical stimulation in intact
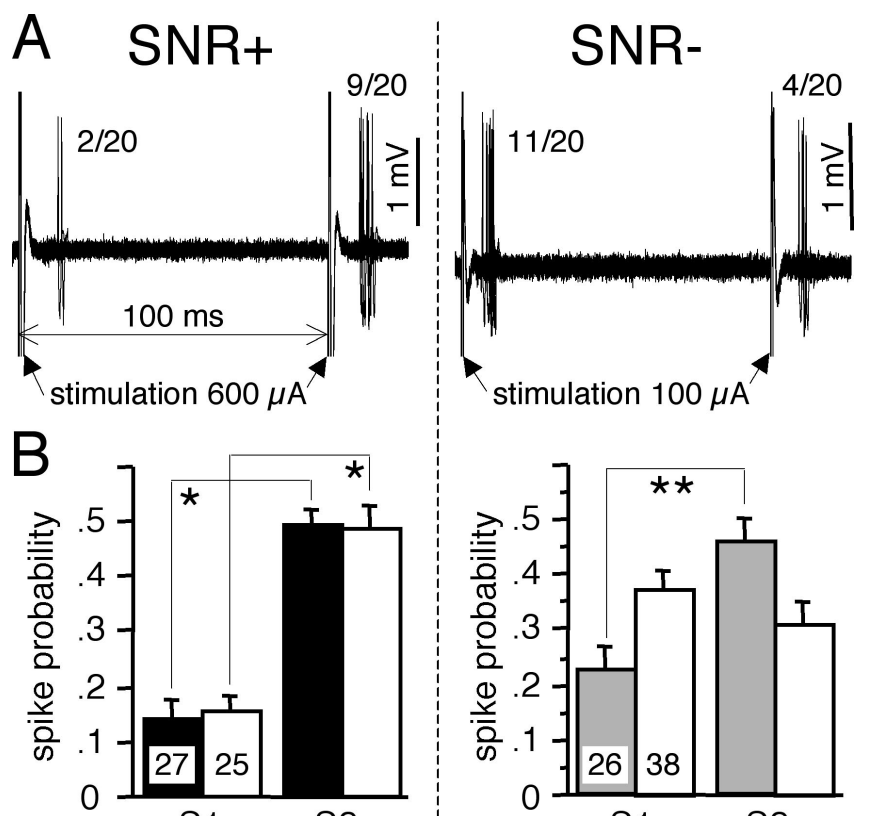

C

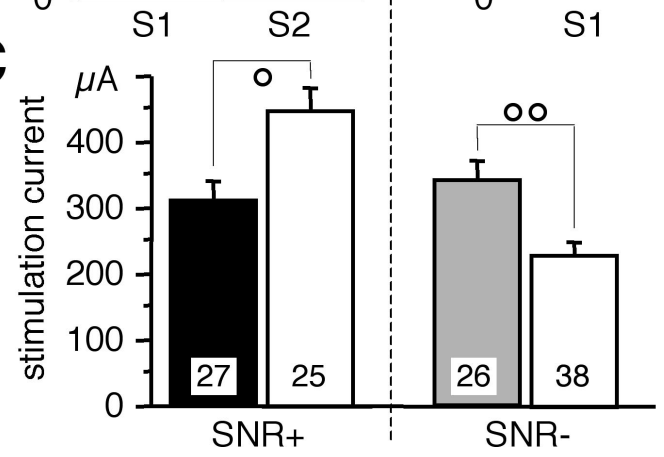

Figure 5. Effect of dopaminergic depletion on the responses of MSNs to pairs of cortical stimulations. A, Pairs of cortical stimulation (100 ms interval) were applied 20 times every $3 \mathrm{~s}$. For each individual MSN, the stimulating current was adjusted by preliminary tests so that the spike response, to either the first or second pulse, reached a probability close to $50 \%$. The 20 consecutive traces recorded from one SNR ${ }^{+}$(left) and one SNR ${ }^{-}$(right) in 6-OHDA-lesioned rats were superimposed. $\boldsymbol{B}$, Discharge probability of SNR ${ }^{+}$(left) and SNR ${ }^{-}$(right) MSNs recorded from sham-operated (filled bars) and from 6-OHDA-lesioned (open bars) rats obtained in the experimental conditions described in $\boldsymbol{A}$. Note that most MSNs were much less responsive to the first pulse in sham-operated rats and that dopaminergic depletion did not affect this pattern regarding SNR ${ }^{+}$MSNs but reversed it regarding SNR ${ }^{-}$MSNs (paired $t$ test, ${ }^{*} p<$ $0.0001 ; * * p=0.007)$. C, Minimal stimulating current that was needed to reach a response probability of $50 \%$ evoked by either the first or second pulse. Bar histograms indicate mean \pm SEM (unpaired $t$ test, ${ }^{\circ} p=0.007 ;{ }^{\circ 0} p=0.001$ ). The number of neurons studied is indicated inside bars. Dopaminergic depletion (open bars) inversely affected the responses of SNR ${ }^{+}$and $\mathrm{SNR}^{-}$neurons.

rats (Fig. 6). Indeed, picrotoxin injection enhanced the response probability (Fig. 6A,B) and increased the variability of the response latency (Fig. $6 C$ ). Under picrotoxin influence, when the response probability was reduced to control level by decreasing the stimulating current (Fig. 6B), the spike response was significantly delayed and the variability of the latency was further enhanced (Fig. 6C).

Because feedforward inhibition by FSIs constrains the time window of the MSN responses to cortical stimulation, we tested whether this function was altered in the DA-depleted striatum. The latency of MSN responses to cortical stimulation was compared in sham-operated rats and in 6-OHDA-lesioned rats (Fig. 7). The lesion enhanced the mean latency in $\mathrm{SNR}^{-}$but not in $\mathrm{SNR}^{+}$neurons (Fig. $7 B$ ). Moreover, the variability of the latency 

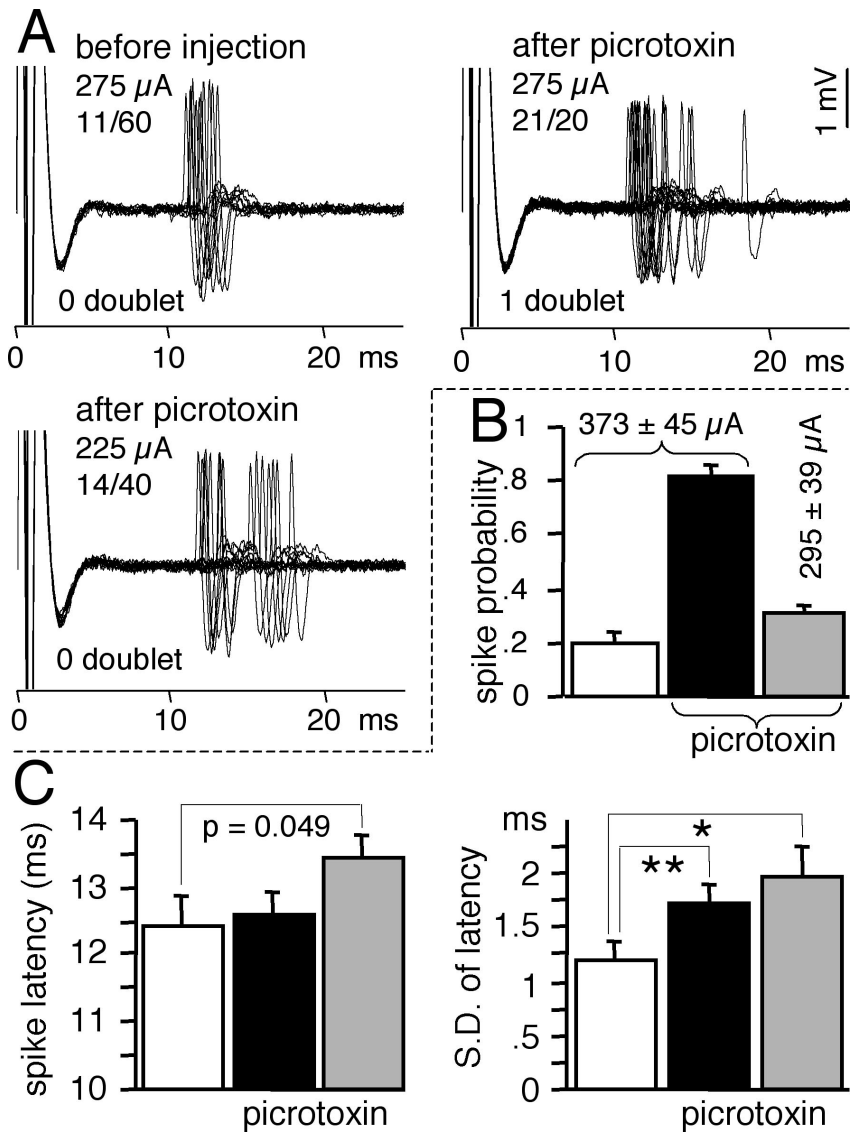

Figure 6. Effect of local picrotoxin injection on the latency of MSN responses to cortical stimulations. Data were obtained from experiments performed in intact rats as reported previously (Mallet et al., 2005). Because picrotoxin similarly affected SNR ${ }^{+}$and SNR ${ }^{-}$MSNs, data from 15 MSNs were pooled. $\boldsymbol{A}$, Superimposition of the spike responses of one MSN before and after picrotoxin. For each individual MSN, the stimulating current was adjusted by preliminary tests so that the spike response to cortical stimulation reached a probability close to $20 \%$. Before picrotoxin injection, the response probability and the latency to spike was recorded from a series of $40-80$ stimulations applied every $3 \mathrm{~s}$, so that at least eight spike responses were obtained. Picrotoxin (1 mm, 20-30 $\mathrm{nl}$ within 6-12 s) was injected by air pressure $150 \mu \mathrm{m}$ above the recorded neuron. After a recovery time of $1 \mathrm{~min}$, the spike response to cortical stimulation was tested, first at the same stimulating current as in control condition and then with a current that was decreased to obtain a response probability similar to control. $\boldsymbol{B}$, As reported previously, picrotoxin dramatically enhanced the response probability (black bar) compared with control (white bar), and a probability similar to control was restored by decreasing the stimulating current (gray bar). C, Mean (left) and variability (right) of the latency of the spike responses before picrotoxin (white bars), after picrotoxin at control current (black bars), and after picrotoxin at decreased current (gray bars). Bar histograms indicate mean $\pm \operatorname{SEM}(n=15$; paired $t$ test, ${ }^{*} p=0.020 ;{ }^{* *} p=0.005$ ). When compared at similar response probabilities, picrotoxin both delayed and scattered spike responses.

was decreased by the lesion in $\mathrm{SNR}^{+}$neurons, and the reverse effect was observed regarding $\mathrm{SNR}^{-}$neurons (Fig. 7B).

\section{Discussion}

Models of Parkinson's disease propose that dopaminergic degeneration disrupts the equilibrium between striatonigral and striatopallidal pathways (Albin et al., 1989; DeLong, 1990; Gerfen, 2000; Hikosaka et al., 2000; Obeso et al., 2000, 2004; Bezard et al., 2001). The expected imbalance in discharge activities was shown here for the first time, to our knowledge. This imbalance has been thought to be directly caused by the lack of dopaminergic receptor stimulation in the parkinsonian striatum, and our study does not exclude this possibility. However, we show here that two novel mechanisms, which are not directly related to this lack,
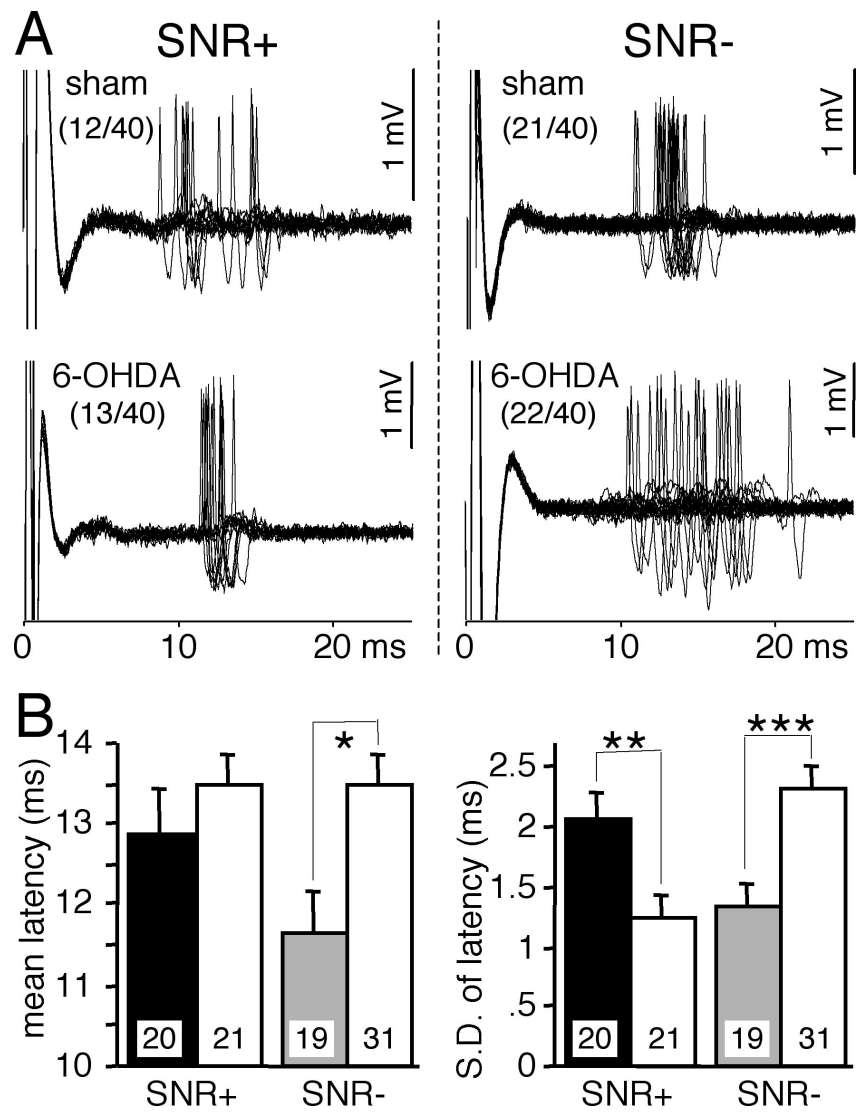

Figure 7. Effect of dopaminergic depletion on the latency of MSN responses to cortical stimulation at similar discharge probability. $\boldsymbol{A}$, Typical examples of spike responses evoked in two $\mathrm{SNR}^{+}$and two SNR ${ }^{-}$MSNs in sham-operated and in 6-OHDA-lesioned rats. Recordings of all spikes (number in parentheses) obtained in each neuron were superimposed. The spike responses of SNR ${ }^{+}$neurons to the first pulse in a pair were collected from experiments depicted in Figure 5. Only neurons exhibiting at least eight spike responses in a series of $40-60$ successive cortical stimulations applied every 3 s were considered. Regarding each SNR ${ }^{-}$neuron, the stimulating current was adjusted by preliminary tests so that the response probability reached $50 \%$ in a series of 40 consecutive single stimulations applied every $3 \mathrm{~s}$. SNR ${ }^{-}$neurons that did not reach this response rate at the maximal stimulation current $(700 \mu \mathrm{A})$ were discarded. $\boldsymbol{B}$, Mean (left) and variability (right) of the latency of the spike responses recorded in shamoperated rats (filled bars) or in 6-OHDA-lesioned rats (open bars) regarding SNR ${ }^{+}$(black bars) and SNR ${ }^{-}$(gray bars) MSNs. Bar histograms indicate mean \pm SEM (unpaired $t$ test, ${ }^{*} p=$ $0.012 ;{ }^{* *} p=0.007 ;{ }^{* * *} p<0.001$ ). The number of neurons tested is indicated inside bars. Dopaminergic depletion enlarged the time window of SNR ${ }^{-}$responses but narrowed that of $\mathrm{SNR}^{+}$responses.

might secondarily contribute to the striatal imbalance. First, specific corticostriatal neurons preferentially innervating striatonigral and striatopallidal neurons exhibited imbalanced activities in the hemi-parkinsonian brain. Second, because FSI spontaneous activity and responsiveness were not altered by dopaminergic depletion, feedforward inhibition of MSNs by FSIs worsened the striatal imbalance.

\section{Dopaminergic depletion imbalances striatonigral and} striatopallidal activities

In intact rats, striatonigral and striatopallidal MSNs exhibited similar responsiveness to cortical stimulation (Mallet et al., 2005). Moreover, most MSN are spontaneously silent, but, on average, striatonigral MSNs tend to be less active than striatopallidal MSNs (Mallet et al., 2005). Several in vivo studies have consistently shown that, on average, the discharge activity of unidentified MSNs is enhanced in 6-OHDA-lesioned rats (Schultz and 
Ungerstedt, 1978; Alloway and Rebec, 1984; Kish et al., 1999; Chen et al., 2001; Tseng et al., 2001). Our data are consistent with these observations because the absolute increase in the spontaneous activity we observed in $\mathrm{SNR}^{-}$neurons greatly exceeded the decrease observed in $\mathrm{SNR}^{+}$neurons. Likewise, a decrease in the threshold current required to obtain cortical responses has also been reported after 6-OHDA lesion (Nisenbaum and Berger, 1992; Florio et al., 1993). It is likely, as shown here, that this increased responsiveness applied only to striatopallidal neurons. The pronounced decrease in the responsiveness of striatonigral MSNs that we observed may have escaped previous investigations because dopaminergic depletion renders these neurons both completely silent and poorly responsive to cortical stimulation. Therefore, dopaminergic depletion inhibits the discharge activity and responsiveness to cortical input of striatonigral neurons and enhances those of striatopallidal neurons. Thus, we directly demonstrate the imbalance in MSN activities hypothesized by classical models of Parkinson's disease.

\section{Corticostriatal neurons are imbalanced by dopaminergic lesion}

Striatonigral and striatopallidal neurons are preferentially innervated by IT-type and PT-type cortical neurons, respectively (Lei et al., 2004). Starting from this seminal study, we used antidromic stimulations, which allowed us to identify corticostriatal neurons. With this approach, identification of IT-type neurons should present a high degree of certainty, whereas that of PT-type neurons should be more cautiously considered. Indeed, the lack of antidromic response evoked from the contralateral striatum may have an instrumental origin. Nevertheless, our approach was selective enough to reveal that dopaminergic lesion imbalanced the activities of both corticostriatal pathways. In particular, the decreased activity of IT-type neurons might contribute to decrease the activity and responsiveness to cortical stimulation of striatonigral MSNs. Indeed, MSN firing only occurs during their depolarization state. The membrane potential achieved during depolarization "is the result of an interaction between a strong depolarizing synaptic conductance" sustained by cortical input and voltage-dependent potassium currents (Wilson and Kawaguchi, 1996). Therefore, the cortical input provided by ITtype neurons might be too weak to generate an efficient depolarization of striatonigral neurons, although intracellular studies are needed to confirm this conclusion. The corticostriatal imbalance cannot directly result from the dopaminergic degeneration because the orofacial motor cortex does not receive any dopaminergic innervation (Bjorklund and Lindvall, 1984). Although the mechanisms that cause the corticostriatal imbalance remain to be determined, our observation points out that the dopaminergic lesion not only alters activities in the basal ganglia but also indirectly affects the whole basal ganglia-thalamo-cortical circuits.

Our observations suggest that cortical imbalance contributes to imbalance activities in the DA-denervated striatum but do not exclude a direct consequence of striatal DA depletion at presynaptic and postsynaptic levels. In fact, striatal DA depletion might actually aggravate the striatal imbalance caused by cortical imbalance. Indeed, in DA-denervated slices, glutamatergic input on striatal neurons is enhanced in a subpopulation of MSNs via a $\mathrm{D}_{2}$-sensitive presynaptic mechanism (Calabresi et al., 1993). However, it remains to be studied whether this enhanced glutamatergic input preferentially affects striatopallidal neurons. Stimulation of $\mathrm{D}_{2}$ postsynaptic receptors selectively decreases the excitability of striatopallidal neurons by inhibiting L-type calcium currents (Hernandez-Lopez et al., 2000). Therefore, the lack of $\mathrm{D}_{2}$ postsynaptic receptor stimulation might also contribute to enhance spontaneous and evoked discharge activities that we observed in striatopallidal neurons recorded in parkinsonian rats. Likewise, the lack of DA stimulation on $\mathrm{D}_{1}$ postsynaptic receptors located on striatonigral neurons might contribute to inhibit striatonigral discharge activities in hemi-parkinsonian rats. Indeed, $\mathrm{D}_{1}$ receptor activation enhances the excitability of MSNs when they are in a depolarized state (Hernandez-Lopez et al., 1997). Finally, striatal DA depletion might also alter $D_{1}$ and $\mathrm{D}_{2}$ receptor modulation of sodium and potassium currents in MSNs (Nicola et al., 2000).

\section{Feedforward inhibition of MSNs by FSIs worsens striatal imbalance}

In vitro studies have shown that FSIs are depolarized by DA via dopaminergic receptors of the $\mathrm{D}_{1} / \mathrm{D}_{5}$ type (Bracci et al., 2002; Centonze et al., 2003). However, we observed that the spontaneous activity and responsiveness of FSIs to cortical stimulation were not significantly altered by dopaminergic depletion. This might be attributable to the fact that our experiments were performed on anesthetized rats, an experimental condition that does not affect the tonic discharge activity of dopaminergic neurons but does depress their bursting activity (Fa et al., 2003). Indeed, the basal extracellular DA level maintained by this tonic activity might be sufficient to stimulate $\mathrm{D}_{2}$ receptors, whereas stimulation of dopaminergic receptors of the $\mathrm{D}_{1}$ type might require a pronounced and phasic increase of extracellular DA induced by the bursting activity (Gonon, 1997; Goto and Grace, 2005). Therefore, in experimental conditions of low bursting activity, the influence of dopamine on FSIs might be undetectable. In line with this interpretation, the chemical stimulation of cortical activity in awake, freely moving rats actually induces an immediateearly gene response in striatal parvalbumin-positive neurons, and this response is significantly attenuated by dopaminergic depletion (Trevitt et al., 2005). FSIs receive massive excitatory inputs from multiple cortical and thalamic areas (Sidibe and Smith, 1999; Ramanathan et al., 2002). The fact that their discharge activity is not affected by the decreased activity of IT-type neurons suggests either that this specific input plays a minor role on FSIs or that unknown mechanisms compensate for this decrease.

The feedforward inhibition of MSNs by FSIs is effective in intact rats because the spike response of FSIs occurs earlier and can be induced by a lower intensity of cortical stimulation than that required for MSNs (Mallet et al., 2005). Dopaminergic depletion did not alter the kinetics and sensitivity of FSI responses and did not shorten the latency of MSN responses. However, it decreased the responsiveness of striatonigral neurons to cortical stimulation and enhanced that of striatopallidal neurons. This should imply that striatonigral MSNs were more strongly inhibited by FSIs, whereas striatopallidal neurons tended to escape from FSI inhibition in the DA-depleted striatum. The feedforward inhibition of MSNs by FSIs can be characterized by a protocol of paired cortical stimulations at $100 \mathrm{~ms}$ time interval. Indeed, because FSIs are far less responsive to the second than to the first pulse, feedforward inhibition more strongly affects the response of MSNs to the first pulse (Mallet et al., 2005). In the DA-denervated striatum, this pattern was still observed regarding striatonigral neurons but was reversed regarding striatopallidal ones. This further suggests that striatopallidal neurons escape, at least partly, from feedforward inhibition.

In vitro studies in the hippocampus and cerebellum have revealed an important function of feedforward inhibition by GABA interneurons: it narrows the time window of the responses trans- 
mitted by principal cells (Pouille and Scanziani, 2001; Mittmann et al., 2005). We showed that FSIs achieve this function in the intact striatum. In the DA-denervated striatum, the time window of the spike responses to cortical stimulation was narrower for striatonigral neurons and wider for striatopallidal neurons compared with sham-operated rats. All of our results suggest that, in the DA-denervated striatum, striatonigral MSNs are more strongly inhibited by FSIs, whereas striatopallidal neurons tend to escape this inhibition. Because FSIs are not directly affected by dopaminergic depletion in our conditions, feedforward inhibition did not cause the imbalance between striatonigral and striatopallidal MSNs, but it did worsen it.

This worsening effect of feedforward inhibition includes an important temporal component. Indeed, in the cerebellum, the temporal precision of spike responses of Purkinje cells is tightly controlled by feedforward inhibition. This control limits the time window for the summation of multiple asynchronous inputs (Mittmann et al., 2005). Likewise, the fact that DA depletion scatters the time window of the response of striatopallidal neurons implies that poorly synchronized cortical inputs should inappropriately summate in these neurons. Conversely, the discharge response of striatonigral neurons to multiple cortical inputs should be further hindered by the fact that the time window of their response is narrowed in the DA-denervated striatum. These temporal disorders might contribute to the pathological oscillations observed in the basal ganglia of parkinsonian patients (Hutchison et al., 2004).

\section{Physiopathological consequences}

Abnormal rhythmic activities in the motor cortex seem to be involved in Parkinson symptoms (Goldberg et al., 2002). Moreover, electrical stimulation of motor cortex reduces motor deficits in 1-methyl-4-phenyl-1,2,3,6-tetrahydropyridine-treated monkeys (Drouot et al., 2004). Our finding that the imbalance of specific corticostriatal neurons might contribute to the striatal imbalance observed after dopaminergic depletion represents a novel contribution to the view that cortical disorders are involved in Parkinson's disease. We also point out the aggravating consequences of feedforward inhibition by FSIs in the DA-denervated striatum. This important observation opens new questions concerning the effects of classical anti-parkinsonian treatments on striatal feedforward inhibition. Answers to these questions might provide the experimental background for the use of enhancers of GABA transmission in combination with classical dopaminergic anti-parkinsonian therapies.

\section{References}

Albin RL, Young AB, Penney JB (1989) The functional anatomy of basal ganglia disorders. Trends Neurosci 12:366-375.

Alloway KD, Rebec GV (1984) Apomorphine-induced inhibition of neostriatal activity is enhanced by lesions induced by 6-hydroxydopamine but not by long-term administration of amphetamine. Neuropharmacology 23:1033-1039.

Bezard E, Brotchie JM, Gross CE (2001) Pathophysiology of levodopainduced dyskinesia: potential for new therapies. Nat Rev Neurosci 2:577-588.

Bjorklund A, Lindvall O (1984) Dopamine-containing systems in CNS. In: Handbook of chemical neuroanatomy (Bjorklund A, Hokfelt T, eds), pp 55-122. Amsterdam: Elsevier.

Bracci E, Centonze D, Bernardi G, Calabresi P (2002) Dopamine excites fast-spiking interneurons in the striatum. J Neurophysiol 87:2190-2194.

Calabresi P, Mercuri NB, Sancesario G, Bernardi G (1993) Electrophysiology of dopamine-denervated striatal neurons. Implications for Parkinson's disease. Brain 116:433-452.

Centonze D, Grande C, Usiello A, Gubellini P, Erbs E, Martin AB, Pisani A,
Tognazzi N, Bernardi G, Moratalla R, Borrelli E, Calabresi P (2003) Receptor subtypes involved in the presynaptic and postsynaptic actions of dopamine on striatal interneurons. J Neurosci 23:6245-6254.

Chen MT, Morales M, Woodward DJ, Hoffer BJ, Janak PH (2001) In vivo extracellular recording of striatal neurons in the awake rat following unilateral 6-hydroxydopamine lesions. Exp Neurol 171:72-83.

Cowan RL, Wilson CJ (1994) Spontaneous firing patterns and axonal projections of single corticostriatal neurons in the rat medial agranular cortex. J Neurophysiol 71:17-32.

DeLong MR (1990) Primate models of movement disorders of basal ganglia origin. Trends Neurosci 13:281-285.

Deniau JM, Menetrey A, Charpier S (1996) The lamellar organization of the rat substantia nigra pars reticulata: segregated patterns of striatal afferents and relationship to the topography of corticostriatal projections. Neuroscience 73:761-781.

Drouot X, Oshino S, Jarraya B, Besret L, Kishima H, Remy P, Dauguet J, Lefaucheur JP, Dolle F, Conde F, Bottlaender M, Peschanski M, Keravel Y, Hantraye P, Palfi S (2004) Functional recovery in a primate model of Parkinson's disease following motor cortex stimulation. Neuron 44:769-778.

Fa M, Mereu G, Ghiglieri V, Meloni A, Salis P, Gessa GL (2003) Electrophysiological and pharmacological characteristics of nigral dopaminergic neurons in the conscious, head-restrained rat. Synapse 48:1-9.

Florio T, Di Loreto S, Cerrito F, Scarnati E (1993) Influence of prelimbic and sensorimotor cortices on striatal neurons in the rat: electrophysiological evidence for converging inputs and the effects of 6-OHDA-induced degeneration of the substantia nigra. Brain Res 619:180-188.

Gerfen CR (2000) Molecular effects of dopamine on striatal-projection pathways. Trends Neurosci 23:S64-S70.

Gerfen CR, Wilson CJ, eds (1996) The basal ganglia. Amsterdam: Elsevier.

Goldberg JA, Boraud T, Maraton S, Haber SN, Vaadia E, Bergman H (2002) Enhanced synchrony among primary motor cortex neurons in the 1-methyl-4-phenyl-1,2,3,6-tetrahydropyridine primate model of Parkinson's disease. J Neurosci 22:4639-4653.

Gonon F (1997) Prolonged and extrasynaptic excitatory action of dopamine mediated by $\mathrm{D}_{1}$ receptors in the rat striatum in vivo. J Neurosci 17:5972-5978.

Goto Y, Grace AA (2005) Dopaminergic modulation of limbic and cortical drive of nucleus accumbens in goal-directed behavior. Nat Neurosci 8:805-812.

Hernandez-Lopez S, Bargas J, Surmeier DJ, Reyes A, Galarraga E (1997) $D_{1}$ receptor activation enhances evoked discharge in neostriatal medium spiny neurons by modulating an L-type $\mathrm{Ca}^{2+}$ conductance. J Neurosci 17:3334-3342.

Hernandez-Lopez S, Tkatch T, Perez-Garci E, Galarraga E, Bargas J, Hamm $\mathrm{H}$, Surmeier DJ (2000) $\mathrm{D}_{2}$ dopamine receptors in striatal medium spiny neurons reduce $\mathrm{L}$-type $\mathrm{Ca}^{2+}$ currents and excitability via a novel PLC $\beta 1-$ $\mathrm{IP}_{3}$-calcineurin signaling cascade. J Neurosci 20:8987-8995.

Hikosaka O, Takikawa Y, Kawagoe R (2000) Role of the basal ganglia in the control of purposive saccadic eye movements. Physiol Rev 80:953-978.

Hutchison WD, Dostrovsky JO, Walters JR, Courtemanche R, Boraud T, Goldberg J, Brown P (2004) Neuronal oscillations in the basal ganglia and movement disorders: evidence from whole animal and human recordings. J Neurosci 24:9240-9243.

Kawaguchi Y (1993) Physiological, morphological, and histochemical characterization of three classes of interneurons in rat neostriatum. J Neurosci 13:4908-4923.

Kawaguchi Y, Wilson CJ, Augood SJ, Emson PC (1995) Striatal interneurones: chemical, physiological and morphological characterization. Trends Neurosci 18:527-535.

Kish LJ, Palmer MR, Gerhardt GA (1999) Multiple single-unit recordings in the striatum of freely moving animals: effects of apomorphine and D-amphetamine in normal and unilateral 6-hydroxydopamine-lesioned rats. Brain Res 833:58-70.

Kita H, Kosaka T, Heizmann CW (1990) Parvalbumin-immunoreactive neurons in the rat neostriatum: a light and electron microscopic study. Brain Res 536:1-15.

Koos T, Tepper JM (1999) Inhibitory control of neostriatal projection neurons by GABAergic interneurons. Nat Neurosci 2:467-472.

Koos T, Tepper JM, Wilson CJ (2004) Comparison of IPSCs evoked by spiny and fast-spiking neurons in the neostriatum. J Neurosci 24:7916-7922. 
Le Moine C, Bloch B (1995) D1 and D2 dopamine receptor gene expression in the rat striatum: sensitive cRNA probes demonstrate prominent segregation of D1 and D2 mRNAs in distinct neuronal populations of the dorsal and ventral striatum. J Comp Neurol 355:418-426.

Lei W, Jiao Y, Del Mar N, Reiner A (2004) Evidence for differential cortical input to direct pathway versus indirect pathway striatal projection neurons in rats. J Neurosci 24:8289-8299.

Mallet N, Le Moine C, Charpier S, Gonon F (2005) Feedforward inhibition of projection neurons by fast spiking GABA interneurons in the rat striatum in vivo. J Neurosci 25:3857-3869.

Mittmann W, Koch U, Hausser M (2005) Feed-forward inhibition shapes the spike output of cerebellar Purkinje cells. J Physiol (Lond) 563:369-378.

Nicola SM, Surmeier DJ, Malenka RC (2000) Dopaminergic modulation of neuronal excitability in the striatum and nucleus accumbens. Annu Rev Neurosci 23:185-215.

Nisenbaum ES, Berger TW (1992) Functionally distinct subpopulations of striatal neurons are differentially regulated by GABAergic and dopaminergic inputs. I. In vivo analysis. Neuroscience 48:561-578.

Obeso JA, Rodriguez-Oroz MC, Rodriguez M, Lanciego JL, Artieda J, Gonzalo N, Olanow CW (2000) Pathophysiology of the basal ganglia in Parkinson's disease. Trends Neurosci 23:S8-S19.

Obeso JA, Rodriguez-Oroz M, Marin C, Alonso F, Zamarbide I, Lanciego JL, Rodriguez-Diaz M (2004) The origin of motor fluctuations in Parkinson's disease: importance of dopaminergic innervation and basal ganglia circuits. Neurology 62:S17-S30.

Plenz D, Kitai ST (1998) Up and down states in striatal medium spiny neurons simultaneously recorded with spontaneous activity in fast-spiking interneurons studied in cortex-striatum-substantia nigra organotypic cultures. J Neurosci 18:266-283.
Pouille F, Scanziani M (2001) Enforcement of temporal fidelity in pyramidal cells by somatic feed-forward inhibition. Science 293:1159-1163.

Ramanathan S, Hanley JJ, Deniau JM, Bolam JP (2002) Synaptic convergence of motor and somatosensory cortical afferents onto GABAergic interneurons in the rat striatum. J Neurosci 22:8158-8169.

Schultz W, Ungerstedt U (1978) Short-term increase and long-term reversion of striatal cell activity after degeneration of the nigrostriatal dopamine system. Exp Brain Res 33:159-171.

Sidibe M, Smith Y (1999) Thalamic inputs to striatal interneurons in monkeys: synaptic organization and co-localization of calcium binding proteins. Neuroscience 89:1189-1208.

Svenningsson P, Le Moine C, Kull B, Sunahara R, Bloch B, Fredholm BB (1997) Cellular expression of adenosine A2A receptor messenger RNA in the rat central nervous system with special reference to dopamine innervated areas. Neuroscience 80:1171-1185.

Svenningsson P, Fourreau L, Bloch B, Fredholm BB, Gonon F, Le Moine C (1999) Opposite tonic modulation of dopamine and adenosine on c-fos gene expression in striatopallidal neurons. Neuroscience 89:827-837.

Trevitt JT, Morrow J, Marshall JF (2005) Dopamine manipulation alters immediate-early gene response of striatal parvalbumin interneurons to cortical stimulation. Brain Res 1035:41-50.

Tseng KY, Kasanetz F, Kargieman L, Riquelme LA, Murer MG (2001) Cortical slow oscillatory activity is reflected in the membrane potential and spike trains of striatal neurons in rats with chronic nigrostriatal lesions. J Neurosci 21:6430-6439.

Wilson CJ, Kawaguchi Y (1996) The origins of two-state spontaneous membrane potential fluctuations of neostriatal spiny neurons. J Neurosci 16 2397-2410. 